\title{
AtMAP65-1 Binds to Tubulin Dimers to Promote Tubulin Assembly
}

\author{
Hua Li, Ming Yuan and Tonglin Mao* \\ State Key Laboratory of Plant Physiology and Biochemistry; Department of Plant Sciences, College of Biological Sciences, \\ China Agricultural University, Beijing 100094, China
}

Received 22 September 2006, Accepted 20 October 2006

In Arabidopsis thaliana, the microtubule-associated protein AtMAP65-1 shows various functions on microtubule dynamics and organizations. However, it is still an open question about whether AtMAP65-1 binds to tubulin dimers and how it regulates microtubule dynamics. In present study, the tubulin-binding activity of AtMAP65-1 was investigated. Pull-down and co-sedimentation experiments demonstrated that AtMAP65-1 bound to tubulin dimers, at a molar ratio of $1: 1$. Cross-linking experiments showed that AtMAP65-1 bound to tubulin dimers by interacting with $\alpha$-tubulin of the tubulin heterodimer. Interfering the bundling effect of AtMAP65-1 by addition of salt and monitoring the tubulin assembly, the experiment results indicated that AtMAP65-1 promoted tubulin assembly by interacting with tubulin dimers. In addition, five truncated versions of AtMAP65-1, namely AtMAP65-1 $\triangle$ N339 (amino acids 340-587); AtMAP65-1 $\Delta$ N494 (amino acids 495-587); AtMAP65-1 340-494 (amino acids 340-494); AtMAP65-1 $\Delta$ C495 (amino acids 1-494) and AtMAP65-1 $\Delta$ C340 (amino acids 1-339), were tested for their binding activities and roles in tubulin polymerization in vitro. Four (AtMAP65-1 $\Delta \mathrm{N339}, \Delta \mathrm{N} 494$, AtMAP65-1 340-494 and $\Delta \mathrm{C495)}$ from the five truncated proteins were able to co-sediment with microtubules, and three (AtMAP65-1 $\Delta$ N339, $\Delta$ N494 and AtMAP65-1 340-494) of them could bind to tubulin dimers in vitro. Among the three truncated proteins, AtMAP65-1 $\triangle \mathrm{N} 339$ showed the greatest activity to promote tubulin polymerization, AtMAP65-1 $\Delta$ N494 exhibited almost the same activity as the full length protein in promoting tubulin assembly, and AtMAP65-1 340-494 had minor activity to promote tubulin assembly. On the contrast,

\footnotetext{
Abbreviations: MAPs, Microtubule-associated proteins; MT, microtubule; PIPES, piperazine-N,N'-bis (2-ethanesulfonic acid; 1,4-piperazinediethanesulfonic acid); EGTA, ethylene glycol-bis-( $\beta$-aminoethyl ether)-N,N,N',N'-tetraacetic acid
}

*To whom correspondence should be addressed.

Tel: 86-10-62731323; Fax: 86-10-62891332

E-mail: mt1524@263.net
AtMAP65-1 $\Delta$ C495, which bound to microtubules but not to tubulin dimers, did not affect tubulin assembly. Our study suggested that AtMAP65-1 might promote tubulin assembly by binding to tubulin dimers in vivo.

Keywords: AtMAP65-1, Arabidopsis, Dynamics, Microtubules, Tubulin

\section{Introduction}

Microtubule associated proteins (MAPs) play a major role in the regulation of microtubule organizations and dynamics. To dissect the mechanisms of MAP's functions on microtubules, it is necessary to examine the regions of MAP's sequences which are responsible for the interaction with microtubules and/or tubulin dimers in detail. Progresses have been made about the functions of various motifs of the MAPs on tubulinbinding in animal cells (Patel et al., 1993; Taylor et al., 2000). It was demonstrated that some MAPs, such as CLIP170, CRMP-2 and stathmin, had tubulin-binding activities (Gachea et al., 2005). These MAPs showed different effects on microtubule assembly and disassembly both in vitro and in vivo (Jourdain et al., 1997; Arnal et al., 2004). Recently, using tubulin affinity chromatography, many tubulin-binding proteins were identified from Arabidopsis cell suspension culture, some of them are plant MAPs (Chuong et al., 2004).

MAP65/PRC1/Ase1 proteins form a family of evolutionarily conserved MAPs. The MAP65 protein family was first identified biochemically in tobacco BY-2 cells, which constitutes 3 or 4 proteins of approximately $65 \mathrm{kDa}$ size (Chang-jie et al., 1993; Smertenko et al., 2000). Two different activities of NtMAP65s in microtubule dynamics have been reported. Although they shares $85 \%$ of sequence identity, the two proteins exert different effects on microtubules dynamics in vitro. NtMAP65-1a enhances microtubule polymerization but NtMAP65-1b does not (Smertenko et al., 2000; Wicker-Planquart et al., 2004). Further result shows that NtMAP65-1a does not bind to tubulin dimers (Chang et al., 2005). 
Nine AtMAP65 genes have been found at Arabidopsis genome database (Hussey et al., 2002). Experiments with the members of this family showed that they bind to microtubules both in vitro and in cells. However, the reports from different labs are controversy about the activities of AtMAP65-1 on microtubule polymerization in vitro (Smertenko et al., 2004; Mao et al., 2005). In addition, detailed biochemical properties or functions of AtMAP65-1 regulation for microtubule polymerization remain to be elucidated.

In present paper, we expressed the full-length and truncations of AtMAP65-1 in bacteria and investigated the properties of these recombinant proteins for microtubule dynamics. Our study demonstrated that full-length of AtMAP65-1 bound to tubulin dimers in vitro. EDC cross-linking analysis showed that AtMAP65-1 bound to $\alpha$-tubulin of the tubulin heterodimer specifically. AtMAP65-1 promoted tubulin assembly by binding to tubulin dimers in vitro. Analysis of the truncated versions of AtMAP65-1 showed that different regions of AtMAP65-1 exhibited different activities in binding to tubulin dimers and tubulin assembly.

\section{Material and methods}

Plasmid and constructs. The full-length cDNA sequence of AtMAP65-1 was obtained and the GST fusion proteins were expressed and purified as descript previously (Mao et al., 2005). The cDNA sequences encoding AtMAP65-1 $\Delta$ C495 (amino acids 495-587 deleted), $\Delta \mathrm{N} 494$ (amino acids 1-494 deleted), $\Delta \mathrm{C} 340$ (amino acids 340-587 deleted), $\Delta \mathrm{N} 339$ (amino acids 1-339 deleted), 340-494 (amino acids 340-494 of AtMAP65-1) were amplified by PCR. The GST fusion proteins were purified with the glutathioneSepharose resin (Amersham Biosciences), according to the manufacturer's protocols.

GST pull-down assays. The purified tubulins were centrifuged at $150,000 \times \mathrm{g}$ at $4^{\circ} \mathrm{C}$ for $20 \mathrm{~min}$ before used. To couple the fusion proteins to the sepharose beads, GST and GST fusion proteins were incubated with glutathione sepharose 4B (Amersham Biosciences), respectively, in the buffer $\mathrm{L}(50 \mathrm{mM}$ Tris- $\mathrm{HCl}, 250 \mathrm{mM} \mathrm{NaCl}$, $1 \mathrm{mM}$ EDTA, $\mathrm{pH} 8.0$ ) in the presence of $0.2 \mathrm{mg} / \mathrm{ml}$ bovine serum albumin (Roche, Germany) for $1 \mathrm{~h}$ at $4^{\circ} \mathrm{C}$. After the fusion-proteincoated beads were washed with buffer $\mathrm{L}, 9 \mu \mathrm{M}$ tubulin was incubated with the beads for $1 \mathrm{~h}$ at $4^{\circ} \mathrm{C}$ in PEM buffer $(100 \mathrm{mM}$ PIPES, $1 \mathrm{mM} \mathrm{MgCl} 2,1 \mathrm{mM}$ EGTA, pH6.9). After the incubation, the beads were washed and re-suspended with $100 \mu$ of $2 \times$ sample buffer. The mixture was boiled for $10 \mathrm{~min}$ to dissociate the proteincomplex from the beads and centrifuged. The supernatant fraction was subject to SDS-PAGE.

Co-sedimentation assay between AtMAP65-1 and tubulin dimers. Samples, containing $5 \mu \mathrm{M}$ AtMAP65-1 and/or $10 \mu \mathrm{M}$ tubulin in the solution (100 mM PIPES, $1 \mathrm{mM}$ EGTA, $1 \mathrm{mM} \mathrm{MgCl}_{2}$ ), respectively, were incubated at $4^{\circ} \mathrm{C}$ for $40 \mathrm{~min}$ and then centrifuged at $350,000 \times \mathrm{g}$ for $10 \mathrm{~min}$. Proteins in supernatants and pellets were analyzed by SDS-PAGE, according to Hamada et al. (2004).
Cross-linking assay. After centrifuged at $200,000 \times \mathrm{g}$ for $15 \mathrm{~min}$ at $4^{\circ} \mathrm{C}$, tubulin and GST-AtMAP65-1 were co-incubated in PEM buffer for $1 \mathrm{~h}$ at $4^{\circ} \mathrm{C}$ with the molar ratio of $1: 1(20 \mu \mathrm{M}$ each) prior to the addition of zero-length cross-linker 1-ethyl-1 [3-(dimethylamino) propyl] carbodiimide (EDC) (Pierce Biotechnology). A final concentration of $2 \mathrm{mM}$ EDC was then added and kept at room temperature for $30 \mathrm{~min}$. The reaction was quenched by adding the same volume of $2 \times$ SDS sample buffer. Samples were analyzed by SDS-PAGE and Western blots probed with a mouse anti- $\alpha$-tubulin monoclonal antibody (Sigma), anti- $\beta$-tubulin monoclonal antibody (Sigma), and anti-AtMAP65-1 (according to Mao et al., 2005) antibody, respectively.

Assays of Microtubule co-sedimentation and Polymerization. These assays were carried out according to the protocol described in Mao et al. (2005).

Fluorescence microscopy. Fluorescent microscopy was carried out as described in Mao et al. (2005), using Zeiss LSM 510 META confocal microscope (Zeiss).

\section{Results}

AtMAP65-1 binds to tubulin and especially interacts with $\alpha$-tubulin. In order to determine whether AtMAP65-1 binds to tubulin, GST pull-down analysis was performed in vitro. The beads coated with GST-AtMAP65-1 fusion protein were incubated with tubulin, and then separated by centrifugation. The proteins binding to the beads were analyzed by SDSPAGE and Western blots. The experiment results showed that GST-AtMAP65-1 bound to tubulin (Fig. 1A, Lane 1), even in the presence of $200 \mathrm{mM} \mathrm{NaCl}$ (Fig. 1A, Lane 2). Western blots probed with anti- $\alpha$-tubulin antibody confirmed the result (Fig. 1B). Negative control with GST protein showed that no interaction between GST protein and tubulin was detected by both SDSPAGE and Western blots (Fig. 1A, Lane 3; 1B, Lane 3).

Co-sedimentation experiments were also performed. $10 \mu \mathrm{M}$ tubulin and $5 \mu \mathrm{M}$ GST-AtMAP65-1 were incubated at $4^{\circ} \mathrm{C}$ for $30 \mathrm{~min}$. GST protein was substituted for GST-AtMAP65-1 as control. In the experiments, tubulin in the solution should be mostly in the form of tubulin dimers due to the low temperature and the absence of GTP. After centrifuged at 350,000 $\times \mathrm{g}$ for $10 \mathrm{~min}$ at $4^{\circ} \mathrm{C}$, the supernatants and pellets were subject to SDS-PAGE, respectively (Fig. 1C). SDS-PAGE analysis showed that when incubated separately most of AtMAP65-1 and tubulin remained in the supernatants and only small amount of AtMAP65-1 and tubulin appeared in pellets after the centrifugation (Fig. 1C, Lanes 1, 2, 3 and 4). However, when incubated together both AtMAP65-1 and tubulin in pellets increased significantly after the centrifugation at the same speed (Fig. 1C, Lanes 8 and 10). Analysis by density scanning of gels with AlphaImager 2200 indicated that AtMAP65-1 was cosedimented with tubulin dimers at a molar ratio of $1: 1$. This result demonstrated that AtMAP65-1 had the capacity in binding to tubulin dimers. 


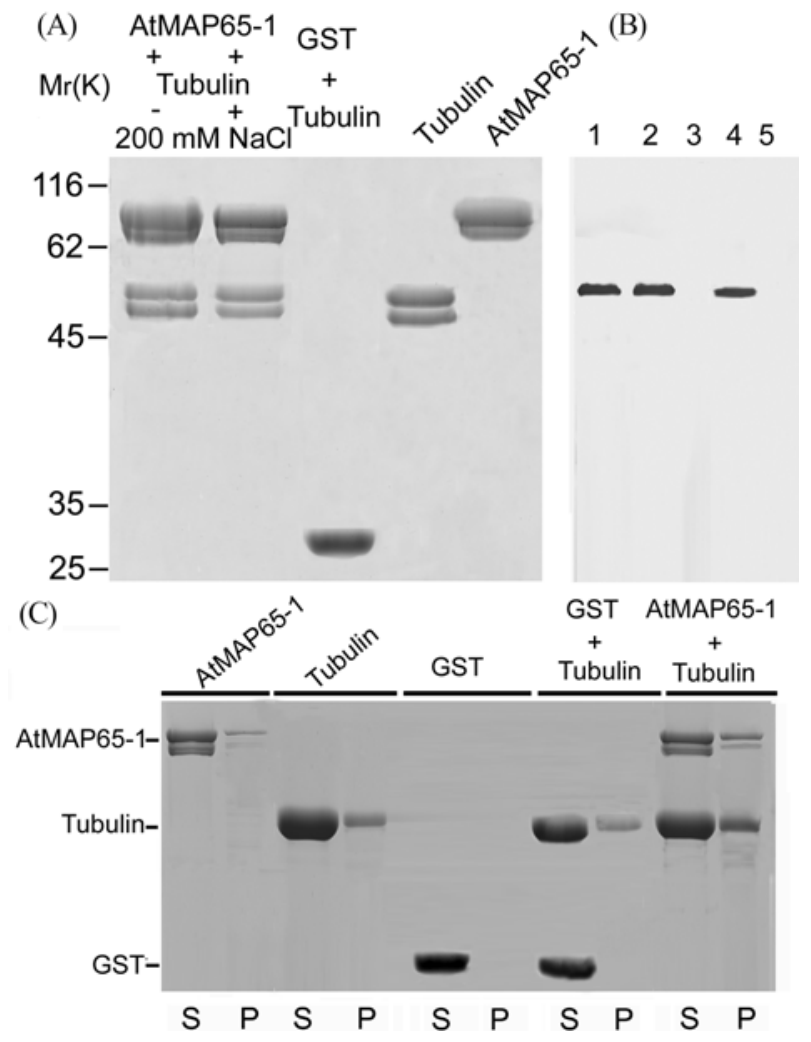

Fig. 1. AtMAP65-1 binds to tubulin heterodimer directly. (A) SDS-PAGE analysis of AtMAP65-1 and tubulin pull-down assay. AtMAP65-1-GST-immobilized beads were incubated with tubulin $(9.0 \mu \mathrm{M})$ in PEM buffer containing $0.2 \mathrm{mg} / \mathrm{ml} \mathrm{BSA}$ for $1 \mathrm{~h}$ at 4 ${ }^{\circ} \mathrm{C}$. The proteins binding to the beads were analyzed by SDSPAGE. Both GST-AtMAP65-1 and tubulin bands were detected on the pull-down beads, or with the addition of $200 \mathrm{mM} \mathrm{NaCl}$. No tubulin band was detected on the pull-down beads when incubated with GST protein-coated beads. Tubulin and GSTAtMAP65-1, respectively, were used as controls. (B) Protein immunoblots with anti- $\alpha$-tubulin antibody, corresponding to A. Tubulin was identified in Lanes 1, 2 and 4. But no tubulin signals were detected in Lanes 3 and 5. (C) Co-sedimentation assay of AtMAP65-1 with tubulin. Samples containing GST-AtMAP65-1 (Lanes 1 and 2), tubulin (Lanes 3 and 4) or both (Lanes 9 and 10) in PEM buffer were incubated and then centrifuged at 350,000 $\times$ g. Supernatants $(\mathrm{S})$ and pellets $(\mathrm{P})$ were subject to SDS-PAGE analysis. GST protein alone (Lanes 5 and 6), and incubated with tubulin (Lanes 7 and 8) were used as controls. GST-AtMAP65-1 mainly presented in supernatant, only a little amount appeared in pellets. Tubulin mainly presented in supernatant too. All of the GST protein was found in the supernatant and none was detected in pellet. When incubated with tubulin, both GST protein and tubulin mainly appeared in supernatant and only a small amount of tubulin was detected in pellet (Lane 8). However, the amount of GST-AtMAP65-1 and tubulin were increased significantly in pellets when they were incubated together.

Furthermore, chemical cross-linking experiment was performed to clarify which tubulin of the heterodimer was responsible for AtMAP65-1 binding. Tubulin dimers and GST-AtMAP65-1
(A)

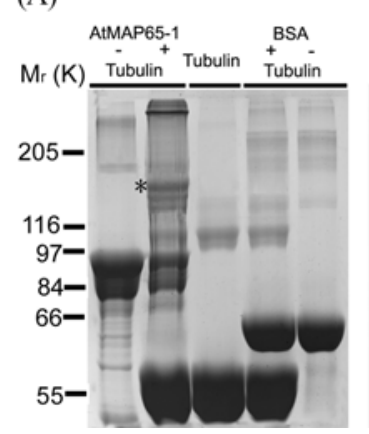

(B)

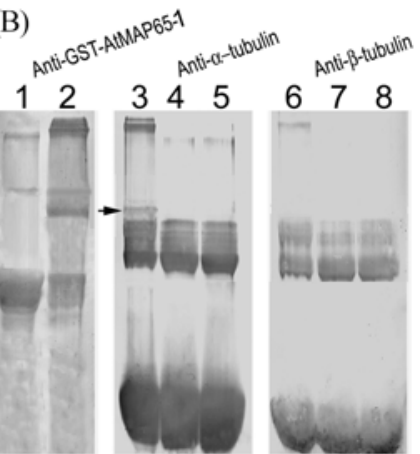

Fig. 2. Cross-linking of AtMAP65-1 with $\alpha$-tubulin by EDC. (A) SDS-PAGE analysis of AtMAP65-1 and tubulin cross-linked assay. GST-AtMAP65-1 and tubulin could be self-cross-linked, respectively (Lanes 1 and 3). When both GST-AtMAP65-1 and tubulin were presented, a specific band was detected (see asterisk) (Lane 2), comparing with Lanes 1 and 3. No interaction was detected between BSA and tubulin (Lane 4). (B) The gel immunoblots were conducted with anti-AtMAP65-1, anti- $\alpha$-tubulin, and anti- $\beta$-tubulin antibodies, respectively. GST-AtMAP65-1 could be dimerized confirmed by probed with anti-AtMAP65-1 antibody (Lane 1). When presented both of GST-AtMAP65-1 and tubulin, the special band could be identified by both antiAtMAP65-1 and anti- $\alpha$-tubulin antibodies, but not anti- $\beta$-tubulin antibody (see arrow) (Lanes 2, 3, 6). Controls by BSA showed that no interactions between tubulin and BSA were detected with both anti- $\alpha$-tubulin and anti- $\beta$-tubulin antibodies (Lanes 5 and 8).

were cross-linked with the zero-length cross linker EDC, analyzed with SDS-PAGE (Fig. 2A), and probed with anti- $\alpha-$ tubulin, anti- $\beta$-tubulin and anti-AtMAP65-1 antibodies, respectively (Fig. 2B). The SDS-PAGE and Western blots analyses showed that both AtMAP65-1 and tubulin could cross-link between themselves (Fig. 2A, Lanes 1, 3; 2B, Lanes 1, 4, 7). However, when both AtMAP65-1 and tubulin dimers presented, a special band was detected after the crosslinking (Fig. 2A, Lane 2). The results of Western blots showed that this band was identified specifically by both anti- $\alpha$ tubulin and anti-AtMAP65-1 antibodies (Fig. 2B, Lanes 2, 3), but not anti- $\beta$-tubulin antibody (Fig. 2B, Lane 6). Negative controls showed that no specific interactions were detected between BSA or BSA and tubulin (Fig. 2A, Lanes 4; 2B, Lanes 5, 8). Therefore, our experiment results demonstrated that AtMAP65-1 bound to tubulin dimers by interaction with $\alpha$-tubulin of the tubulin heterodimer.

AtMAP65-1 may promote microtubule polymerization by interacting with tubulin. In order to gain insight into the mechanism for AtMAP65-1 to promote tubulin polymerization, analysis of tubulin polymerization in the presence of AtMAP65-1 was performed. According to the experiments above, the interaction of AtMAP65-1 and tubulin was not significantly interfered when $\mathrm{NaCl}$ was presented at concentrations below $200 \mathrm{mM}$. Therefore, $120 \mathrm{mM} \mathrm{NaCl}$ was chosen to strip AtMAP65-1 from microtubules, but not tubulins. Confocal 

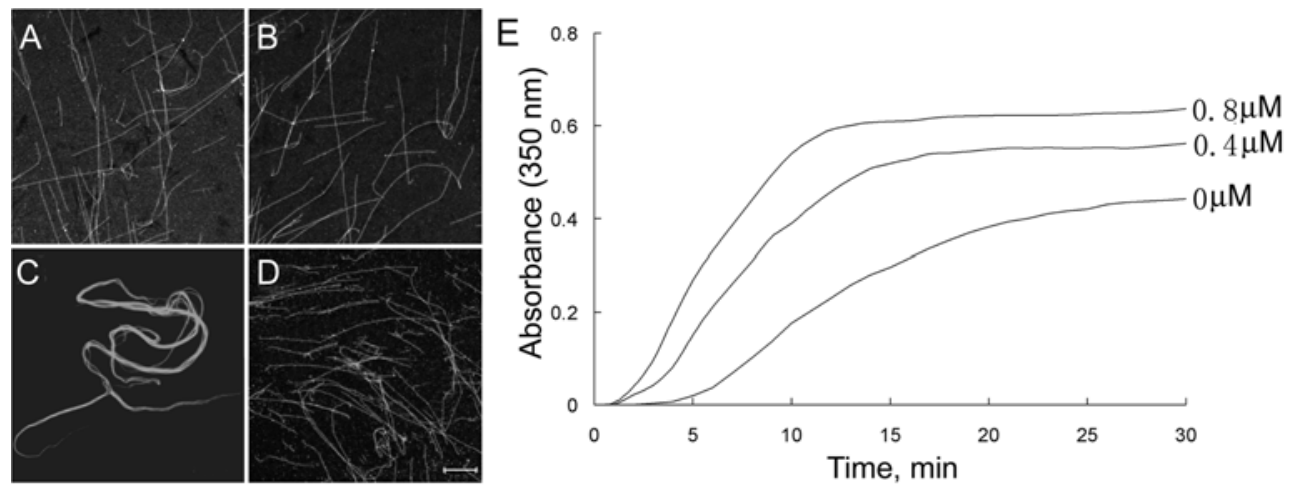

Fig. 3. AtMAP65-1 promotes tubulin assembly without bundling microtubules. Confocal images showed that tubulin assembled in the solution of $20 \mu \mathrm{M}$ NHS-rhodamine labeled tubulin (A), or with the addition of $120 \mathrm{mM} \mathrm{NaCl}$ (B). Large microtubule bundles formed in the presence of AtMAP65-1 (C). However, only single microtubules were observed in the presence of $120 \mathrm{mM} \mathrm{NaCl}$ (D). Further monitoring the time course of polymerization of tubulin, with the addition of $120 \mathrm{mM} \mathrm{NaCl}$, showed that the turbidity curve of tubulin assembly significantly increased in the presence of 0.4 or $0.8 \mu \mathrm{M}$ AtMAP65-1 (E). Scale bar $=10 \mu \mathrm{m}$ for fluorescent images.

microscopy observation showed that tubulins assembled in a pattern of single microtubules in PEM buffer containing 120 $\mathrm{mM} \mathrm{NaCl}$ (Fig. 3B). On the other hand, large bundles of microtubules formed in the presence of AtMAP65-1 (Fig. 3C) loosened into single microtubules when $120 \mathrm{mM} \mathrm{NaCl}$ was added (Fig. 3D). Therefore, addition of $120 \mathrm{mM} \mathrm{NaCl}$ prevented microtubule bundling but not tubulin polymerization in the presence of AtMAP65-1.

By monitoring the turbidity of tubulin suspension at $350 \mathrm{~nm}$, the processes of microtubule polymerization and nucleation in vitro were recorded quantitatively, in PEM buffer containing $120 \mathrm{mM} \mathrm{NaCl}$. In a $20 \mu \mathrm{M}$ tubulin solution, the tubulin polymerization reached a steady state in approximately 25 min (Fig. 3E). When $0.8 \mu \mathrm{M}$ AtMAP65-1 was added, the absorbance value increased more than two folds at its steady state, comparing with the tubulin polymerization in the absence of AtMAP65-1, indicating that more tubulins were polymerized into microtubules (Fig. 3E). In addition, the time to reach the steady state was shortened to less than $15 \mathrm{~min}$. Addition with $0.4 \mu \mathrm{M}$ AtMAP65-1 had similar effect on tubulin polymerization, although not as dramatic as that in the presence of $0.8 \mu \mathrm{M}$ AtMAP65-1 (Fig. 3E). Because no microtubule bundles could form in the presence of $120 \mathrm{mM}$ $\mathrm{NaCl}$, the observation indicated that AtMAP65-1 promoted tubulin polymerization in vitro largely due to the interaction with tubulin dimers, but not due to microtubule bundling and stabilizing activities.

Activities of the different regions of AtMAP65-1 on the regulation of microtubule dynamics. Since AtMAP65-1 has the activities of binding to tubulin and to microtubules, we are interested in which domains of the protein sequence are responsible for regulation the dynamics of tubulin assembly.

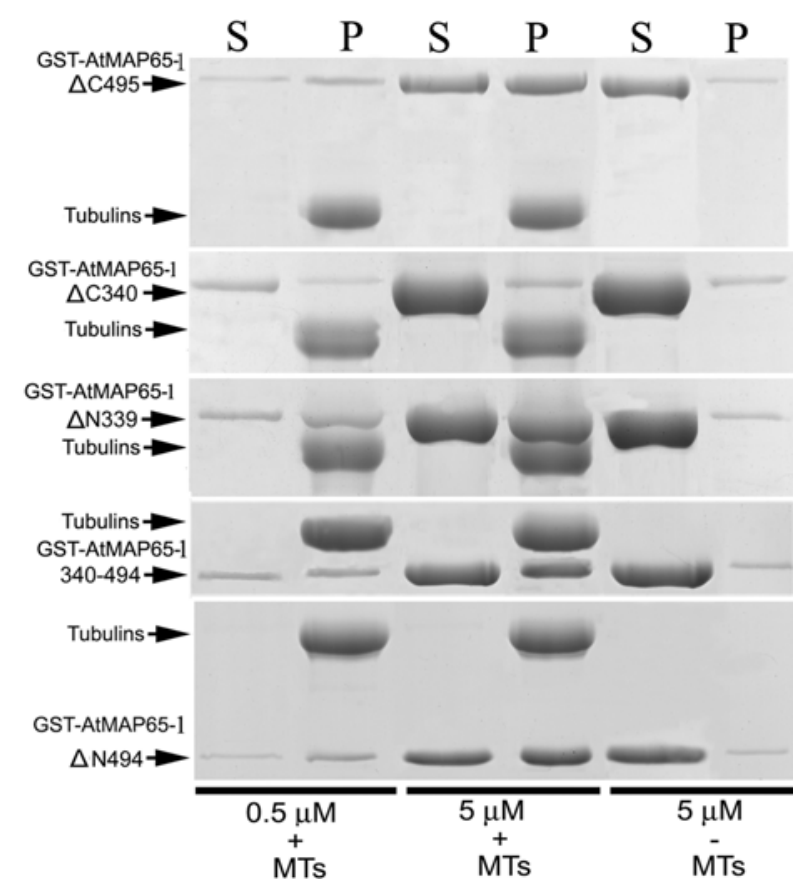

Fig. 4. The fusion proteins of AtMAP65-1 fragments co-sediment with microtubules. $5 \mu \mathrm{M}$ fusion proteins of GST-AtMAP65-1 $\Delta \mathrm{C} 495, \Delta \mathrm{N} 339,340-494, \Delta \mathrm{N} 494$, and $\Delta \mathrm{C} 340$ were incubated with or without $5 \mu \mathrm{M}$ microtubules, respectively. The solutions were then centrifuged, and the supernatant and pellets were subject to SDS-PAGE analysis. In the absence of microtubules, all the fragments of AtMAP65-1 were mainly in the supernatant (Lane 5). However, after incubated with microtubules, the fractions of GST-AtMAP65-1 $\Delta \mathrm{C} 495, \Delta \mathrm{N} 339,340-494, \Delta \mathrm{N} 494$ in the supernatant decreased (Lane 3), while increased in the pellets, respectively (Lane 4). However, GST-AtMAP65-1 $\Delta$ C340 remained in the supernatant even in the presence of microtubules (Lane 4). 


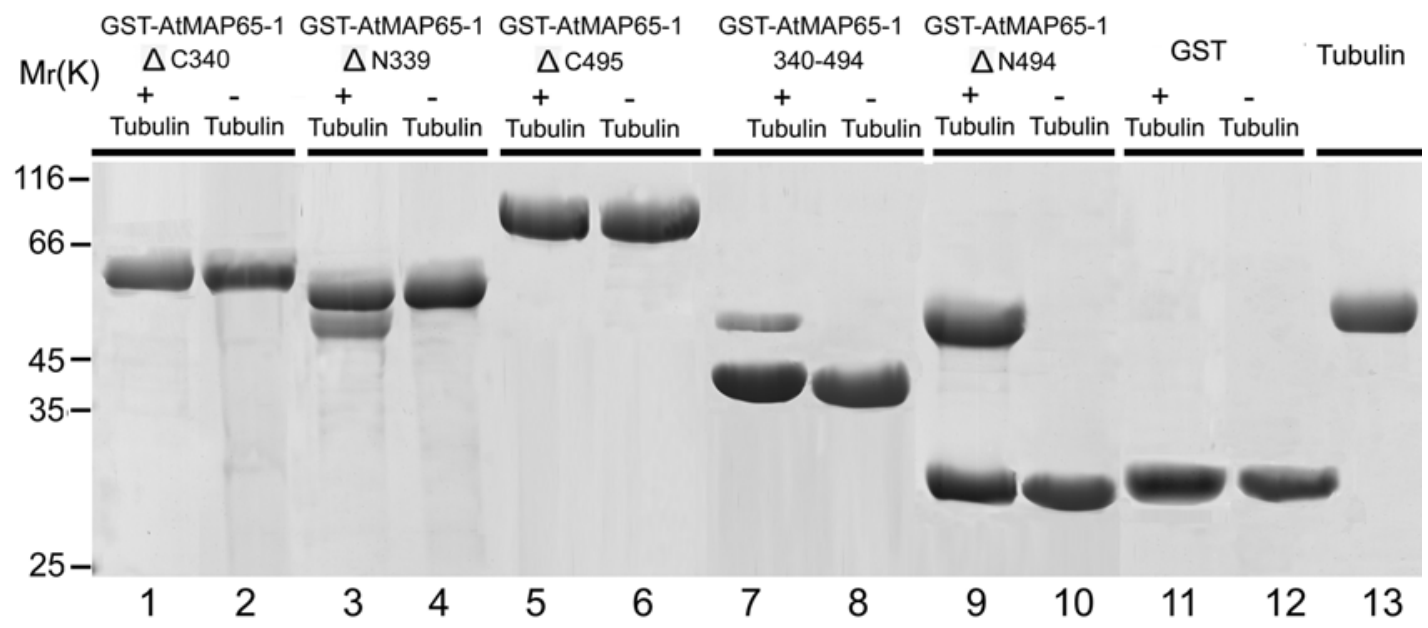

Fig. 5. The fusion proteins of AtMAP65-1 fragments bind to tubulin. The fragments of AtMAP65-1-GST-immobilized beads were incubated with tubulin $(9.0 \mu \mathrm{M})$ in PEM buffer. The beads were separated and the proteins on the beads were analyzed by SDS-PAGE. Tubulin bound to GST-AtMAP65-1 $\Delta$ N339, 340-494 and $\Delta$ N494, respectively (Lanes 3, 7 and 9). However, no binding of tubulin was detected with GST-AtMAP65-1 $\Delta \mathrm{C} 340$ and $\Delta \mathrm{C} 495$ (Lanes 1 and 5). GST protein was used as a control.

Five truncated versions of AtMAP65-1, namely AtMAP65-1 $\Delta \mathrm{N} 339$ (amino acids 340-587); AtMAP65-1 $\Delta \mathrm{N} 494$ (amino acids 495-587); AtMAP65-1 340-494 (amino acids 340-494); AtMAP65-1 $\Delta$ C495 (amino acids 1-494) and AtMAP65-1 $\Delta$ C340 (amino acids 1-339) were chosen according to the alignment of AtMAP65s and Smertenko et al. (2004). The GST-fusion proteins of AtMAP65-1 fragments were purified and used for the biochemical analysis in vitro.

Co-sedimentation experiments were performed to analyze the binding activities for those five truncated proteins. The results showed that $\Delta \mathrm{N} 339$, AtMAP65-1 340-494, $\Delta \mathrm{N} 494$ and $\triangle \mathrm{C} 495$ were co-sedimented with microtubules, but $\Delta \mathrm{C} 340 \mathrm{did}$ not (Fig. 4). The results of $\Delta$ N494 and AtMAP65-1 340-494 were consistent with the previous report (Smertenko et al., 2004). And the results also assured us that the GST fusion partner did not affect the binding activity of these fragments of AtMAP65-1.

Furthermore, we analyzed the activities of different regions of AtMAP65-1 in binding to tubulin and tubulin assembly. The GST pull-down experiments were conducted with those truncated proteins. The experiment results showed that $\triangle \mathrm{N} 339$, AtMAP65-1 340-494 and $\Delta \mathrm{N} 494$ bound to tubulin (Fig. 5, Lanes 3, 7, 9). Among them, $\Delta \mathrm{N} 339$ and $\Delta \mathrm{N} 494$ bound to tubulin more strongly than AtMAP65-1 340-494, judged by scanning the gel (Fig. 5, Lanes 3, 9, 7). However, $\Delta \mathrm{C} 495$ bound to microtubules, but not to tubulin (Fig. 5, Lane 5). So there probably exits different binding sites of AtMAP65-1 for microtubules and tubulin. $\triangle \mathrm{C} 340$ bound to neither microtubules nor tubulin (Fig. 5, Lane 1).

Furthermore, the effect of the truncated proteins on tubulin polymerization was investigated. The results of co-sedimentation experiments showed that the amount of tubulin in pellets was increased with the addition of $\triangle \mathrm{N} 339, \Delta \mathrm{N} 494$ and AtMAP65$1340-494$, comparing with that in the absence of those
AtMAP65-1 fragments (Fig. 6A, B, C). Although $\Delta \mathrm{C} 495$ could bind to microtubules, it did not affect its polymerization (Fig. 6D). Among these truncated proteins, $\Delta \mathrm{N} 339$ had the strongest activity in promoting microtubule polymerization shown by increased amount of microtubules in the pellet (Fig. $6 \mathrm{~A}, \mathrm{E})$. The quantity of microtubules increased over $50 \%$ in the presence of $\Delta \mathrm{N} 339$ than the control. Addition of $\Delta \mathrm{N} 494$ resulted in over $25 \%$ increase of the quantity of microtubules than the control, with an activity similar with full length of AtMAP65-1 as previously reported (Mao et al., 2005) (Fig. 6B, E). However, AtMAP65-1 340-494 had only a minor effect on tubulin polymerization (Fig. 6C, E).

To assess whether these truncated fragments of AtMAP651 have activities in tubulin polymerization by binding to tubulin, the turbidity assay was conducted. The results showed that $\Delta \mathrm{N} 339$ shortened the nucleation time of tubulin assembly dramatically, from $180 \mathrm{~s}$ in control to almost $60 \mathrm{~s}$ in the presence of $1 \mu \mathrm{M}$ AtMAP65-1 $\Delta \mathrm{N} 339$ (Fig. 7A). Addition of $4 \mu \mathrm{M}$ AtMAP65-1 340-494 reduced the nucleation time from $180 \mathrm{~s}$ to $90 \mathrm{~s}$ (Fig. 7B). On the other hand, $\Delta \mathrm{N} 494$ shortened the time from $180 \mathrm{~s}$ to $70 \mathrm{~s}$ (Fig. 7C). So we concluded that $\Delta \mathrm{N} 339, \Delta \mathrm{N} 494$ and AtMAP65-1 340-494 could all bind to tubulin and increased the rate of tubulin assembly.

\section{Discussion}

AtMAP65-1 proteins promote tubulin assembly by interaction with tubulin dimers. Three MAP65 proteins have been identified from tobacco BY-2 suspension cultured cells and carrot suspension cells (Chang-Jie and Sonobe, 1993; Chan et al., 1996). However, NtMAP65-1a and NtMAP65-1b, despite their high degree of homology, have different characteristics on microtubule dynamics (Smertenko 

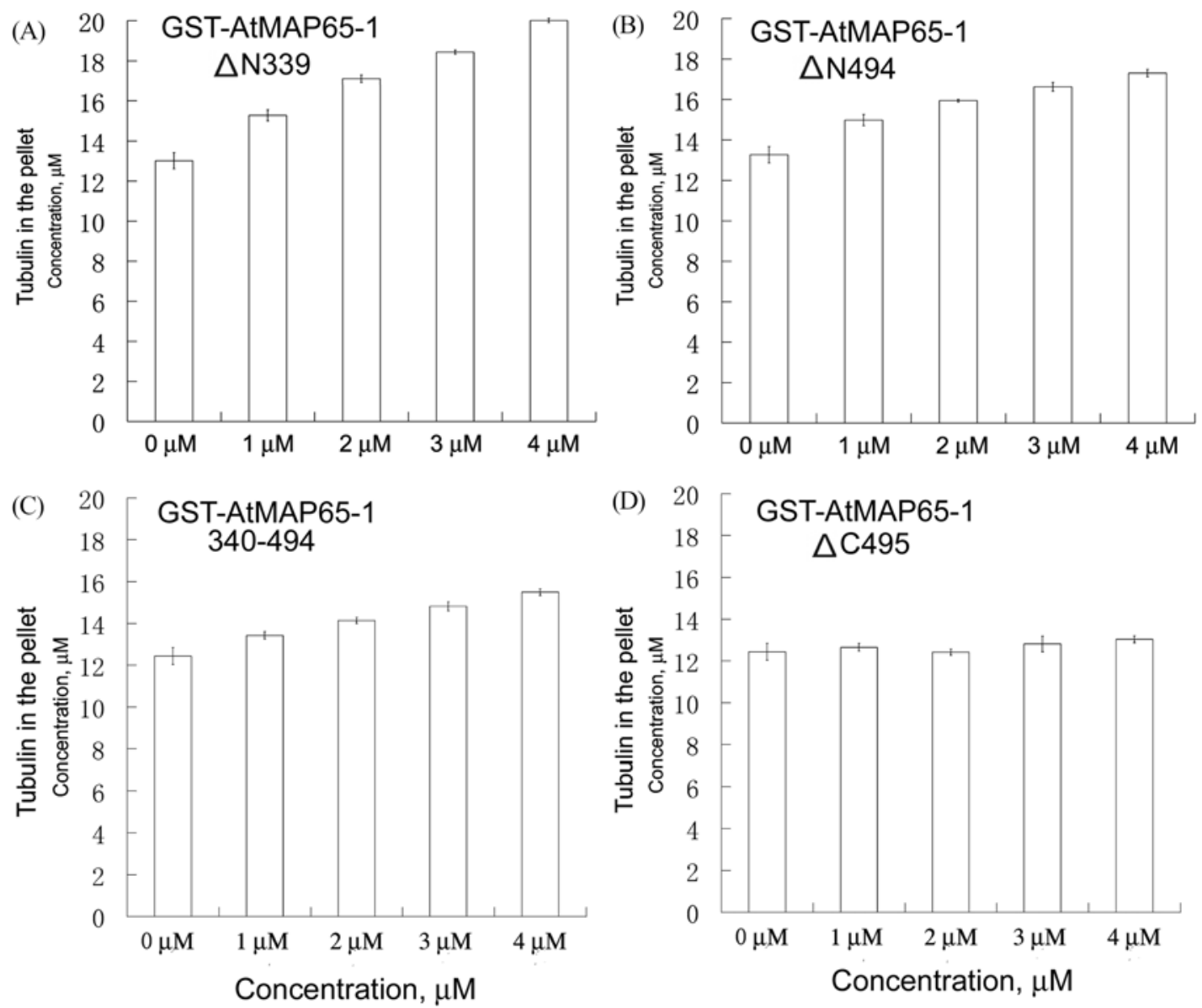

(F)
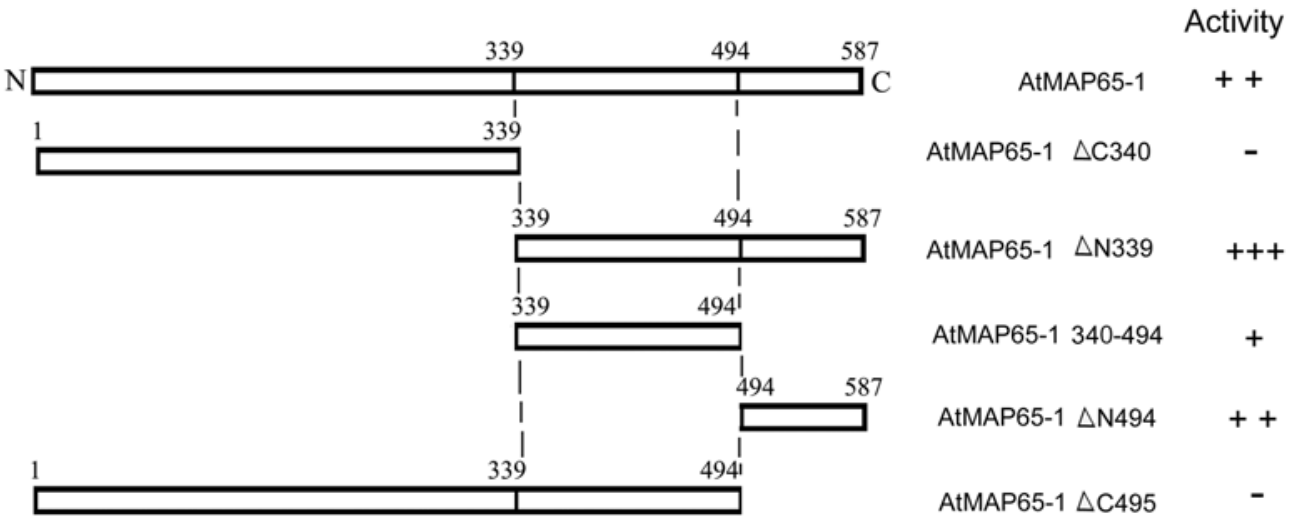

Fig. 6. Analysis of the activities of AtMAP65-1 fragments in promoting tubulin polymerization. Tubulin polymerization was performed in the presence of various concentrations of AtMAP65-1 fragments. Amount of tubulin sedimented and the gels were analyzed by density scanning. Microtubules increased in a concentration dependent manner in the presence of AtMAP65-1 $\triangle$ N339 (A), AtMAP65-1 $\Delta \mathrm{N} 494$ (B), and AtMAP65-1 340-494 (C). However, no obvious change was found in the presence of AtMAP65-1 $\Delta$ C495 (D). At the concentration of $4 \mu \mathrm{M}$, approximately $52 \%$ increase of microtubules in the presence of AtMAP65-1 $\Delta \mathrm{N} 339,30 \%$ in the presence of AtMAP65-1 $\triangle$ N494, and 20\% in the presence of AtMAP65-1 340-494 were measured. E. The relative activity of these AtMAP65-1 fragments on tubulin assembly. +++ indicates more than $50 \%$ increase of the amount of microtubules; ++ indicates over $25 \%$ increase of the amount of microtubules; + indicates less than $25 \%$ increase of the amount of microtubules; - indicates no significant change.

et al., 2000). NtMAP65-1a promotes microtubule assembly but does not bind to tubulin (Smertenko et al., 2000; Chang et al., 2005). NtMAP65-1b bundles microtubules but does not affect tubulin assembly (Wicker-Planquart et al., 2004).

The sequence identity between AtMAP65-1 and NtMAP651a is about $73 \%$ (analyzed by DNAMAN 4.0 software), 

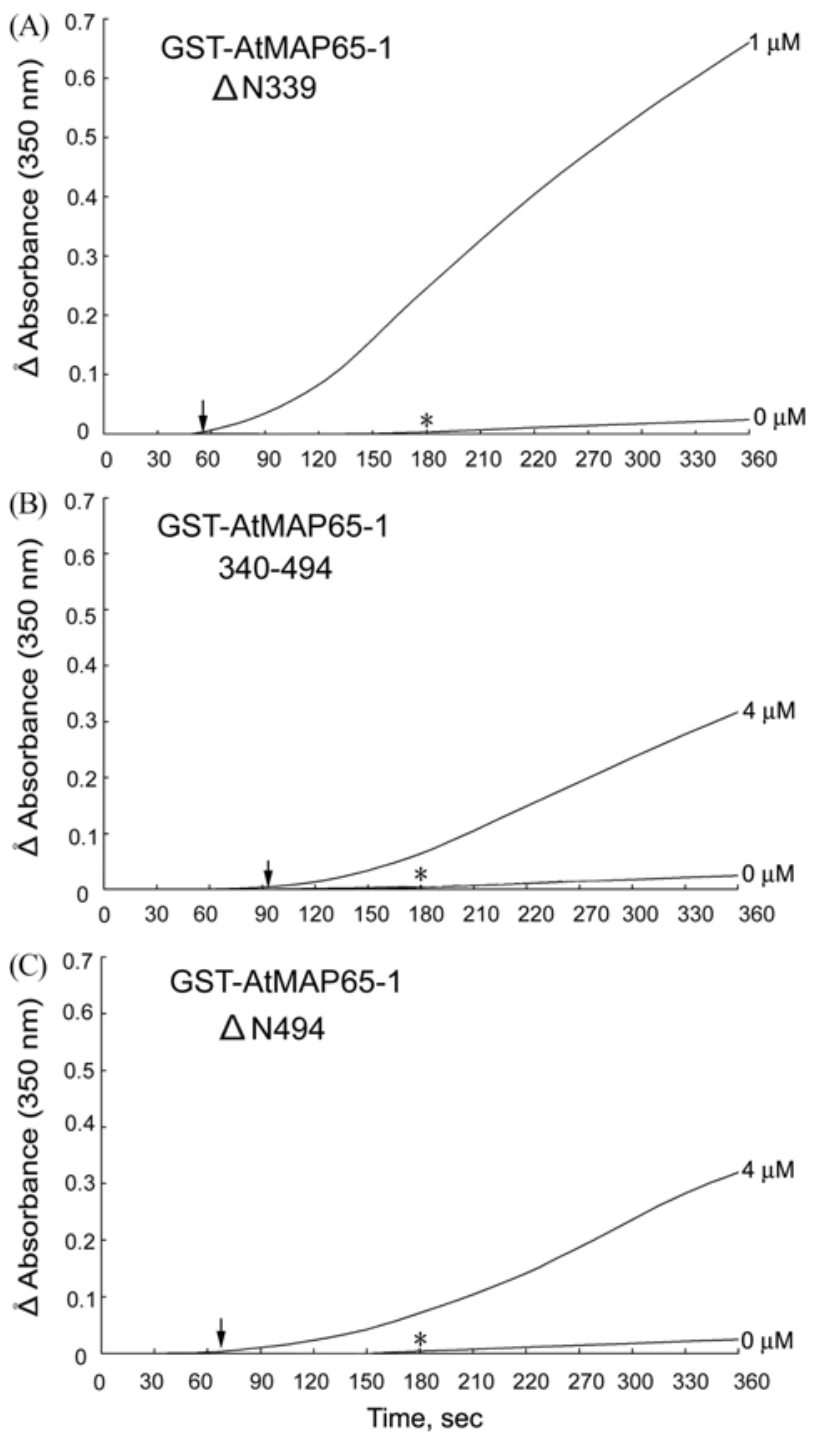

Fig. 7. The fusion proteins of AtMAP65-1 fragments shortened the nucleation time of tubulin assembly. The lag time of tubulin assembly was about $180 \mathrm{sec}$ in the absence of the fusion proteins (see asterisk). In the presence of $1 \mu \mathrm{M}$ AtMAP65-1 $\Delta \mathrm{N} 339$, or 4 $\mu \mathrm{M} 340-494$ and $\Delta \mathrm{N} 494$, however, the lag time was reduced to $60 \mathrm{sec}(\mathrm{A}), 90 \mathrm{sec}(\mathrm{B})$ or $70 \mathrm{sec}$ (C), respectively (see the arrows).

which is less than the identity between NtMAP65-1a and NtMAP65-1b (85\%). Therefore, although AtMAP65-1 is closely related to NtMAP65-1a, they probably have different characteristics on the relationship with microtubules and tubulin (Hussey et al., 2002; Mao et al., 2005).

In the present study, five regions of amino acid sequences of AtMAP65-1 were chosen for analysis. Biochemical experiment data suggested that three regions (AtMAP65-1 $\Delta \mathrm{N} 339$, 340494 and $\Delta \mathrm{N} 494$ ) shorten the nucleation time and promote tubulin assembly. GST pull-down analysis showed that these three regions also bind to tubulin dimers. On the other hand, AtMAP65-1 $\Delta$ C495, which binds to microtubules but not to tubulin dimers, had no effect on tubulin polymerization. These results indicate that AtMAP65-1 promotes tubulin assembly by interacting with tubulin dimers.

The exact mechanism of AtMAP65-1 promoting tubulin assembly by interacting with tubulin dimers is still unknown. Some MAPs both in animal cells and in plant cells have now been identified to promote tubulin polymerization by binding to tubulin dimers directly, such as MAP200 and XMAP215 (Fukata et al., 2002; Hamada et al., 2004). The main mechanism to be considered is that these MAPs, which have more than one binding site for tubulin, may form complexes with tubulin. And the complexes mostly functions as MT-nucleating complexes to accelerate the nucleating process of tubulin assembly (Spittle et al., 2000; Cassimeris et al., 2001; Hamada et al., 2004). According to our biochemical analysis and Smertenko et al. (2004), AtMAP65-1 may be in the states of dimerization in the solution. We hypothesize that the dimers of AtMAP65-1 may provide seeds with binding sites for tubulin dimers to add to, and form a relative big complex. The complex composed of AtMAP65-1 and tubulin dimers may be used as nuclei for tubulin assembly. A large number of short microtubules are observed by confocal microscopy at the early stage of tubulin assembly in the presence of AtMAP65-1 (data not shown). It is suggested that these complexes, formed during the early phase of tubulin assembly, may serve as microtubule-nucleating sites to accelerate the tubulin assembly.

AtMAP65-1 $\triangle$ N494 plays an important role in regulation dynamics of microtubule. The C-terminus of AtMAP65-1, which is responsible for binding to microtubule, shows the strongest activities for promoting tubulin assembly among the five regions. The same phenomenon is also observed for Cytoplasmic Linker Protein CLIP-170 which is a microtubule binding protein. The microtubule binding domain (named $\mathrm{H}_{2}$ ) of CLIP-170 may stimulate microtubule nucleation by binding to tubulin oligomers and forming ring-like complexes (Arnal et al., 2004). AtMAP65-1 340-494 and $\Delta$ N494 both bind to tubulin, indicating that there are two tubulin-binding sites. The C-terminus of AtMAP65-1 also contains two microtubule binding sites as previously reported (Smertenko et al., 2004). AtMAP65-1 $\Delta \mathrm{C} 495$, however, only binds to microtubule but not to tubulin. These results demonstrated that the tubulin-binding site of AtMAP65-1 340-494 is concealed by the N-terminus but the microtubule binding site is not affected. Therefore there are probably different binding domains for tubulin dimers or microtubules in the amino acid sequence of AtMAP65-1. Thus, among the tubulin-binding domains on the full length of AtMAP65-1 there is probably only one available, which lies in the region of amino acids 495-587.

AtMAP65-1 340-494 is regarded as conserved sequences among the AtMAP65s family and plays important role in binding to microtubule. AtMAP65-1 $\Delta$ N494, however, is a most diverse region and its sequence identity with other members of AtMAP65s family is less than $20 \%$, except 
AtMAP65-6 and AtMAP65-2. Our results indicate that AtMAP65-1 $\Delta \mathrm{N} 494$ is probably the major region for the binding to tubulin dimers among the amino acid sequence of AtMAP65-1. The activity of AtMAP65-1 $\triangle$ N494 on dynamics of microtubule is identical with the full length of AtMAP65-1, which also demonstrates that the region for the AtMAP65-1 is more important to regulate tubulin assembly.

Acknowledgment We thank Dr. Bo Liu (Section of Plant Biology, University of California, Davis, California) for useful discussion and critically reading the manuscript. This research was supported by the National Key Basic Research Project of China (2006CB100101 to M.Y.) and National Natural Science Foundation of China (30421002, 30370707, and 30570925 to M.Y.; 30600312 to T.M.)

\section{References}

Arnal, I., Heichette, C., Diamantopoulos, G. S. and Chrètien, D. (2004) CLIP-170/tubulin-curved oligomers coassemble at microtubule ends and promote rescues. Curr. Biol. 14, 20862095.

Cassimeris, L., Gard, D., Tran, P. T. and Erickson, H. P. (2001) XMAP215 is a long thin molecule that does not increase microtubule stiffness. J. Cell Sci. 114, 3025-3033.

Chan, J., Rutten, T. and Lloyd, C. (1996) Isolation of microtubuleassociated proteins from carrot cytoskeletons: a $120 \mathrm{kDa}$ map decorates all four microtubule arrays and the nucleus. Plant $J$. 10, 251-259.

Chang, H. -Y., Smertenko, A. P., Igarashi, H., Dixon, D. P. and Hussey, P. J. (2005) Dynamic interaction of NtMAP65-1a with microtubules in vivo. J. Cell Sci. 118, 3195-3201.

Chang-Jie, J. and Sonobe, S. (1993) Identification and preliminary characterization of a $65 \mathrm{kDa}$ higher-plant microtubuleassociated protein. J. Cell Sci. 105, 891-901.

Chuong, S. D., Good, A. G., Taylor, G. J., Freeman, M. C., Moorhead, G. B. and Muench, D. G. (2004) Large-scale identification of tubulin-binding proteins provides insight on subcellular trafficking, metabolic channeling, and signaling in plant cells. Mol. Cell. Proteomics 3, 970-983.

Fukata, Y., Itoh, T. J., Kimura, T., Ménager, C., Nishimura, T., Shiromizu, T., Watanabe, H., Inagaki, N., Iwamatsu, A., Hotani, H. and Kaibuchi, K. (2002) CRMP-2 binds to tubulin heterodimers to promote microtubule assembly. Nat. Cell Biol.
4, 583-591.

Gachea, V., Louwagieb, M., Garinb, J., Caudrona, N., Lafanecherea, L. and Valirona, Odile. (2005) Identification of proteins binding the native tubulin dimer. Biochem. Biophys. Res. Commun. 327, 35-42.

Hamada,T., Igarashi, H., Itoh, T. J., Shimmen, T. and Sonobe, S. (2004) Characterization of a $200 \mathrm{kDa}$ microtubule-associated protein of tobacco BY-2 cells, a member of the XMAP215/ MOR1 family. Plant Cell Physiol. 45, 1233-1242.

Hussey, P. J., Hawkins, T. J., Igarashi, H., Kaloriti, D. and Smertenko A. (2002) The plant cytoskeleton: recent advances in the study of the plant microtubule-associated proteins MAP65, MAP-190 and the Xenopus MAP215-like protein, MOR1. Plant Mol. Biol. 50, 915-924.

Jourdain, L., Curmi, P., Sobel, A., Pantaloni, D. and Carlier, M. F. (1997) Stathmin: A tubulin-sequestering protein which forms a ternary T2S complex with two tubulin molecules. Biochemistry 36, 10817-10821.

Mao, T. L., Jin, L. F., Li, H., Liu, B. and Yuan, M. (2005) Two Microtubule-Associated Proteins of the Arabidopsis MAP65 Family Function Differently on Microtubules. Plant Physiol. 138, 654-662.

Patel, N., Thierry-Miegt, D. and Mancillas, J. (1993) Cloning by insertional mutagenesis of a cDNA encoding Caenorhabditis elegans kinesin heavy chain. Proc. Natl. Acad. Sci. USA 90, 9181-9185.

Smertenko, A. P., Saleh, N., Igarashi, H., Mori, H., Hauser-Hahn, I., Jiang, C. J. Sonobe, S., Lloyd, C. W. and Hussey, P. J. (2000) A new class of microtubule-associated proteins in plants. Nat. Cell Biol. 2, 750-753.

Smertenko, A. P., Chang, H. -Yu, Wagner, V., Kaloriti, D., Fenyk, S., Sonobe, S., Lloyd, C., Hauser, M. T. and Hussey, P. J. (2004) The arabidopsis microtubule-associated protein AtMAP65-1: Molecular analysis of its microtubule bundling activity. Plant Cell 16, 2035-2047.

Spittle, C., Charrasse, S., Larroque, C. and Cassimeris, L. (2000) The interaction of TOGp with microtubules and tubulin. $J$. Biol. Chem. 275, 20748-20753.

Taylor, K. R., Holzer, A. K., Bazan, J. F., Walsh, C. A. and Gleeson, J. G. (2000) Patient mutations in doublecortin define a repeated tubulin-binding domain. J. Biol. Chem. 44, 3444234450 .

Wicker-Planquart, C., Stoppin-Mellet, V., Blanchoin, L. and Vantard, M. (2004) Interactions of tobacco microtubuleassociated protein MAP65-1b with microtubules. Plant J. 39, 126-134. 